\title{
Observations of Detailed Structure in the Solar Wind at 1 AU with STEREO/HI-2
}

\author{
C. E. DeForest \\ Department of Space Studies, Southwest Research Institute, Boulder, CO 80302 \\ deforest@boulder.swri.edu \\ T. A. Howard \\ Department of Space Studies, Southwest Research Institute, Boulder, CO 80302 \\ howard@boulder.swri.edu \\ S. J. Tappin \\ National Solar Observatory, Sunspot, NM 88349 \\ jtappin@nso.edu
}

\begin{abstract}
We present images of solar wind electron density structures at distances of 1 A.U., extracted from the STEREO/HI-2 data. Collecting the images requires separating the Thomson-scattered signal from the other background/foreground sources that are $10^{3}$ times brighter. Using a combination of techniques, we are able to generate calibrated imaging data of the solar wind with sensitivity of a few $\times 10^{-17} \mathrm{~B}_{\odot}$, compared to the background signal of a few $\times 10^{-13} \mathrm{~B}_{\odot}$, using only the STEREO/HI-2 Level 1 data as input. These images reveal detailed spatial structure in CMEs and the solar wind at projected solar distances in excess of $1 \mathrm{AU}$, at the instrumental motion-blur resolution limit of $1^{\circ}-3^{\circ}$. CME features visible in the newly reprocessed data from December 2008 include leading-edge pileup, interior voids, filamentary structure, and rear cusps. "Quiet" solar wind features include $\mathrm{V}$ shaped structures centered on the heliospheric current sheet, plasmoids, and "puffs" that correspond to the density fluctuations observed insitu. We compare many of these structures with in-situ features detected near 1 AU. The reprocessed data demonstrate that it is possible to perform detailed structural analyses of heliospheric features with visible light imagery, at distances from the Sun of at least 1 AU.
\end{abstract}

Subject headings: Sun: coronal mass ejections (CMEs), solar-terrestrial relationsSolar System: interplanetary medium-methods: data analysis 


\section{Introduction}

The last decade has seen the emergence of a new class of white light imaging instruments, heliospheric imagers, that combine precise photometric wide-field imaging with extremely deep baffles. Reviews of the capabilities of heliospheric imagers have been written by Harrison et al. (2009) and by Howard (2011).

Heliospheric imagers are in principle capable of detecting and tracking both large- and small-scale transients in the solar wind by collecting and imaging sunlight that is scattered directly off of free electrons in interplanetary space, just as coronagraphs capture the K-corona in the vicinity of the Sun. The first proof-of-concept observations of heliospheric transients were demonstrated with the Helios zodiacal light instrument (Richter et al. 1982; Jackson et al. 1985) and the first wide-field dedicated heliospheric imaging instruments were the Solar Mass Ejection Imager (SMEI), launched in 2003 on the Coriolis spacecraft (Eyles et al. 2003) and the STEREO mission Heliospheric Imagers (HIs), launched in 2006 (Eyles et al. 2009). These instruments have proven to be effective for observing solar wind transient phenomena such as coronal mass ejections (e.g. Howard et al. 2006; Harrison et al. 2008; Webb et al. 2009), corotating interaction regions (e.g. Sheelev et al. 2008b; Rouillard et al. 2008; Tappin \& Howard 2009a), and solar wind "puffs" (e.g. Rouillard et al. 2010) and "blobs" (e.g. Sheeley et al. 2009; Sheeley and Rouillard 2010).

Small, non-CME structure (puffs and blobs) have been observed in the solar wind in the outer corona (e.g. Sheeley et al. 1997), by in-situ spacecraft (e.g. Schwenn et al. 1990; Phillips et al. 1995), using interplanetary scintillation (e.g. Rickett \& Coles 1991), and by the STEREO/HI-1 instruments (e.g. Clover et al. 2010). Quantitative, detailed imaging of these structures has remained elusive at elongation angles above $40^{\circ}$ (in the $S T E R E O / \mathrm{HI}-2$ field of view).

The greatest challenge in heliospheric imaging is signal extraction of the faint Thomson scattering signal from the far brighter foreground and background signals. Large CMEs have intensities (at $45^{\circ}$ solar elongation) of order $10^{-14} \mathrm{~B}_{\odot}$, and the faintest remotely tracked solar wind features (puffs) have intensities of the order of a few $\times 10^{-16} \mathrm{~B}_{\odot}$ (Rouillard et al. 2010; Tappin \& Howard 2009a). ( $1 \mathrm{~B}_{\odot}$ is a solar brightness unit $\sim$ the surface brightness of the solar photosphere). Tappin \& Howard (2009a) have demonstrated that careful analysis of differenced elongation-vs-time images ("J-maps" or "J-plots") can detect features with brightnesses of a few $\times 10^{-16} \mathrm{~B}_{\odot}$, and Rouillard et al. (2010) has performed simlar J-plot analyses of small transient structures propagating with the ambient wind and becoming entrained in a CIR. Both groups used the structure of the J-plot imagery to reveal morphology of features with signal strength at or below the noise level imposed by the background star field in their processed data. To our knowledge no group has yet produced clear 2-D imagery of evolving 
wind features in HI-2 at comparable or greater sensitivity.

These faint signals are detected by STEREO/HI against a background dominated by three principal sources: instrumental stray light, which forms a fixed pattern on the detector; zodiacal light (which we also refer to as " $\mathrm{F}$ corona") at the few $\times 10^{-13} \mathrm{~B}_{\odot}$ level that varies only slowly as the spacecraft orbits the Sun; the background starfield, which is extremely spiky $\left(10^{-14} \mathrm{~B}_{\odot}\right.$ for a 10 th magnitude star in the HI-2A instrument) and drifts with the orbital motion of the spacecraft (Eyles et al. 2003); and small artifacts, reminiscent of cosmic rays or dust, that are visible in many images despite on-board processing that removes most cosmic ray impacts. SMEI is also subjected to saturation from magnetospheric particles, auroral glow, and moonlight. The stray light pattern in STEREO/HI is weak compared to the $\mathrm{F}$ corona.

In the present paper, we describe and demonstrate a technique by which the HI- 2 data can be background-subtracted to reveal solar wind structures throughout the field with residual noise levels of $\sim 3 \times 10^{-17} \mathrm{~B}_{\odot}$ in $1^{\circ}$ square patches of image, within a factor of three of the photon counting noise level (which is $\sim 10^{-17} \mathrm{~B}_{\odot}$ against a background of $10^{-13} \mathrm{~B}_{\odot}$ in the standard HI-2 data set, when averaged over a $1^{\circ}$ square patch).

We have reprocessed HI-2A data across a time period of several days surrounding a well-documented CME that occurred in December 2008. The reprocessed data have a noise floor lower than has been available in previous analyses, and reveal rich detail in the solar wind and CME structure, that has been previously inaccessible.

Along with the CME we identify several solar wind transient features, including the CME itself, a forerunner, a filament, and some small transient structures that may correspond to "puffs" and "blobs" observed by Rouillard et al. (2010) and Sheeley et al. (2009) respectively.

Because the technique does not require difference subtraction, we are also able to identify cavity regions surrounding the CME event itself that have been undetectable with previous analytical techniques. Making J-plots from the resulting cleaned movie shows striking correspondence between the apparent arrival times of the observed heliospheric features at Earth, and the detection of similar density structures by the Wind spacecraft.

We conclude with discussions of the limitations of image processing for extracting solar wind heliospheric image data, of physical implications of high quality imaging of transients in the solar wind, and planned work to exploit the newly available data sets. 


\section{Instrument and Event}

We consider the outer heliospheric imager (HI-2A) on board the STEREO-A ("ahead")

spacecraft (Eyles et al. 2009; Howard et al. 2008; Kaiser et al. 2008). HI consists of two cameras, HI-1 normally observes across an elongation range of $4^{\circ}$ to $24^{\circ}$ and HI- 2 from $19^{\circ}$ to $89^{\circ}$, both centered on the line formed by the image-projected ecliptic plane. STEREO was launched so that its angular separation from the Sun-Earth line increased at a rate of $\sim 22.5^{\circ}$ per year. During the time period of interest (December 2008), STEREO-A was $42^{\circ}$ away from the Sun-Earth line and at a radial distance of 0.97 AU from the Sun. HI-2 operates on a 2 hour cadence, summing many individual camera frames into 7,000 second "macro-exposures" on board the spacecraft. The macro-exposures are despiked on-board in the temporal direction to remove cosmic rays and similar artifacts before summing. The camera has an active image planes of $2048 \times 2048$ pixels but is summed $2 \times 2$ before downlink, so that reduced images are $1024 \times 1024$ pixels in size.

We chose to focus on HI-2A alone rather than a joint study with HI-2A and HI-2B (on the "behind" spacecraft) because the starfield in HI-2B images is more difficult to isolate than from HI-2A, due to an apparent defocus seen in the HI-2B images. That defocus is smaller than the expected motion blur we describe $\left(1^{\circ}-3^{\circ}\right.$ depending on feature speed), but large enough to require slightly different techniques for starfield subtraction than are described here.

The event of primary interest was a CME that erupted from the Sun on 12 December 2008 and reached the HI-2 field of view the following day. It is generally regarded as the first geoeffective CME observed during the STEREO mission and has been documented by Davis et al. (2009), Liu et al. (2010) and Lugaz et al. (2010). Our observational coverage includes the time period from 11-21 December, which includes the pre- and post-CME solar wind in this region. We set out to extract and observe whatever structure we could from the event as it propagated outward.

\section{Processing}

We removed background from the HI-2 images in stages. Like other unpolarized coronagraphs such as $\mathrm{SOHO} / \mathrm{LASCO}$, the HI-2 images include a strong image component from dust (the zodiacal light or "F corona"), which cannot in principle be separated from the fixed, smooth bulk component of the desired Thomson-scattered ("K-corona") signal. Because the $\mathrm{F}$ corona is nearly constant, it can be separated from dynamic transients in the $\mathrm{K}$ coronal signal by tracking and subtracting the minimum brightness in any single pixel on the focal 
plane over an interval long compared to the crossing time of the features of interest. This yields images of the "excess density" due to transient features. A major complication for HI-2 is the background starfield. Unlike coronagraph data, both the F and $\mathrm{K}$ coronal signals in the HI-2 field are faint compared to the bright stars in the starfield. Further, the starfield has different motion characteristics than the F corona because of spacecraft orbital motion, and is extremely spiky, stretching the limits of pattern identification and rectification.

Because the Thomson signal is so faint, additional steps are required to remove secondorder effects not visible in less challenging data, which requires two additional filtration steps. Noise sources are summarized in Table 1. Two bright planets were visible in the field of view - Earth and Venus - and they saturated the detector, leaving top-to-bottom saturated streaks that damaged some of the later filtering steps. We cropped the field of view of our observation to eliminate them.

We began our analysis with STEREO-supplied Level 1 data, which are linearly calibrated (no nonlinear calibration applied) in normalized digitizer counts per second (DN $\mathrm{s}^{-1}$ ). An example frame is given in Figure 1, which has been square-root scaled to show the dominant features in the data: bright stars interspersed with the F corona at exposure levels of a few tens of $\mathrm{DN} \mathrm{s}^{-1}$. The processing includes five major steps: stationary background removal; celestial background removal (including cross-image distortion measurement); residual F corona removal; moving-feature filtration in the Fourier plane; and conversion back to focal plane coordinates. The $\mathrm{K}$ coronal signal is not visible at this brightness scale, but is of order $0.02 \mathrm{DN} \mathrm{s}^{-1}$ compared to the scale of $25 \mathrm{DN} \mathrm{s}^{-1}$.

\subsection{Stationary background removal}

The F corona and stray light signals are both approximately stationary on the image plane. We do not attempt to separate them, but remove both with the same image processing step. We identified the stationary background by simple stacking of the complete 11 day data set. We treated each pixel as a statistical population of brightnesses, and examined the

Table 1: Characteristics of background and noise sources in the STEREO/HI-2 data

\begin{tabular}{|c|c|c|}
\hline Background source & Motion & Amplitude $\left(\mathrm{DN} \mathrm{s}^{-1}\right)$ \\
\hline \hline Stray light & stationary & up to 30 at bright edge \\
\hline F corona & approx. stationary & up to 30 \\
\hline Starfield & moves $1^{\circ} /$ day & up to 20 \\
\hline Galaxy & moves $1^{\circ} /$ day & up to 20 \\
\hline
\end{tabular}




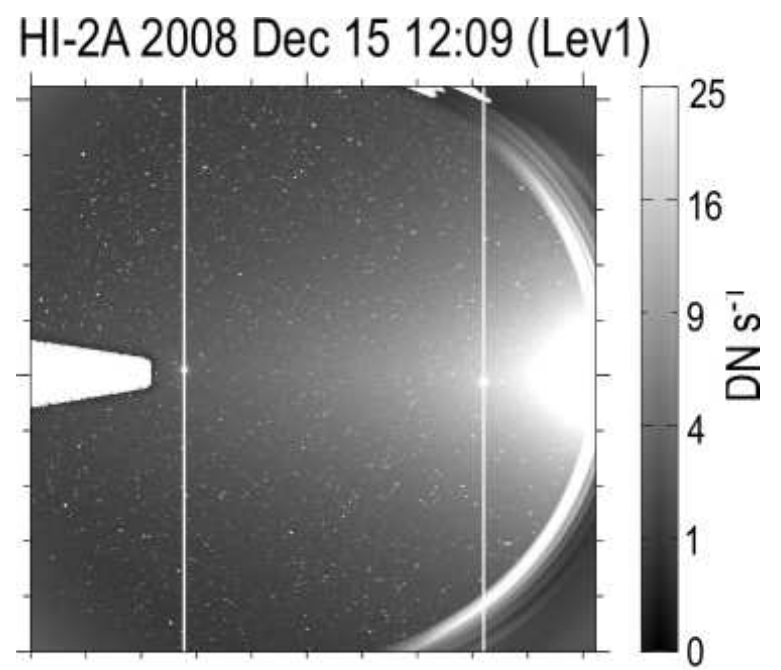

Fig. 1. - Unprocessed STEREO/HI-2 Level 1 image at 0-25 DN s ${ }^{-1}$ (square root scaled to increase dynamic range), obtained on 15 December 2008 at 12:09 UT (2 hour accumulation). The Sun is to the right, but well outside the image, and the coordinates are in units of image pixels. Dominant features include the F corona, starfield, and CCD artifacts including the two planets Earth (left) and Venus (right). The planets saturate the CCD causing "bleeding" of charge in the vertical direction and defining the width of the usable field of view for our analysis. The $\mathrm{K}$ coronal signal of interest has an amplitude of $0.02 \mathrm{DN} \mathrm{s}^{-1}$, 1000 times fainter. 
faintest brightness value from each pixel. Data dropouts are set by the STEREO pipeline to have negative numbers. For the sorting, we treated these numbers as large, so that the faintest valid values of each pixel could be considered. The faintest and brightest value images for the data set spanning 11-21 December 2008 in HI-2a are shown in Figure 2. The faintest-value image contains primarily $\mathrm{F}$ corona and stray light. The brightest-value image on the right of Figure 2 clearly shows the starfield as a set of streaks: as each bright star passes across the field of view over the data set, it sets the brightest value of that pixel. The optics distortion function is visible as a variation in the characteristic length and angle of the streaks across the field.

Measuring the $\mathrm{F}$ corona and stray light requires slightly more care than simply extracting the minimum value. Three important artifacts remain: overexposure streaks from planets and other bright features; residual streaking from the starfield; and statistical noise-induced errors from the $\mathrm{F}$ corona. The first two can be combatted with in-image filtering; the latter is more problematic. Figure 3 shows the minimum value image, unsharp-masked to highlight these remaining structures that are present at the $\pm 0.1 \mathrm{DN} \mathrm{s}^{-1}$ level. The exposure artifacts can be removed by using a low-percentile image (we chose the 5th percentile in pixel brightness) at some cost in additional starfield artifacts. The starfield can be further reduced with spatial median filtration, and we use a $5 \times 5$ median filter on our fixed background image. This technique is similar to that used used by the STEREO/HI-2 official pipeline, which involves taking a per-pixel average of the lowest quartile of values in an 11 day window.

The speckling toward the right in Figure 3 is more problematic than the other two error sources in the fixed pattern image, and we attribute it to variations in the noise level of different detector bins on the CCD, coupled with the skewed sample imposed by a minimum-value measurements. The Level 1 data are linearly flat-field corrected and blur compensated for exposure during readout, but it is of course not possible to eliminate all detection noise, and the data value at each pixel is the sum of the image plus samples of independent random variables from photon counting and the inherent fixed-pattern noise of the CCD. The minimum-value operation across an ensemble of raw images necessarily produces a skewed sample of these detection noise sources. The two sources introduce two visually distinct signatures. The first is due to the fixed pattern noise of the CCD and appears as "speckle" in the brightest portion of the image (center-right). The second is due to skewed sampling of the photon noise from the F corona itself. It yields both a brightness-dependent level of "snow" in Figure 3 and a systematic underestimation of the background brightness. It may in principle be possible to correct for this underestimation, by characterizing the independent statistics of the fixed pattern noise and photon counting noise.

A nice compromise between the rabbit hole of independent statistical treatment of noise 


\section{Dec 11-21 min value}

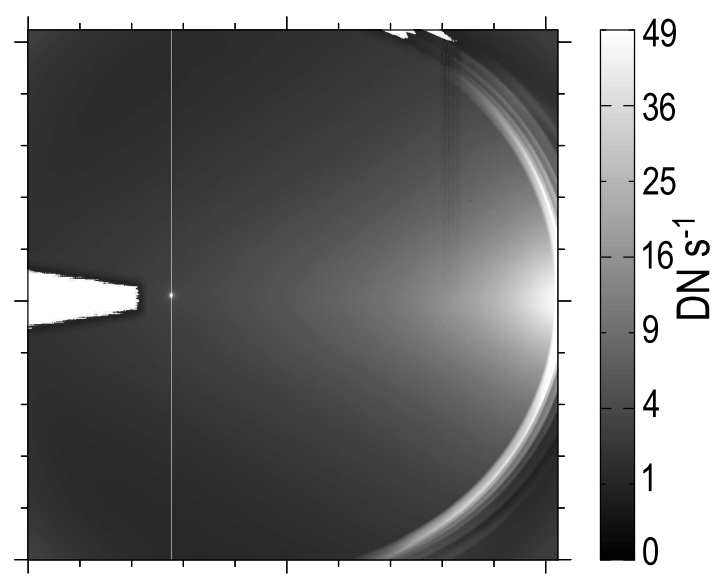

\section{Dec 11-21 max value}

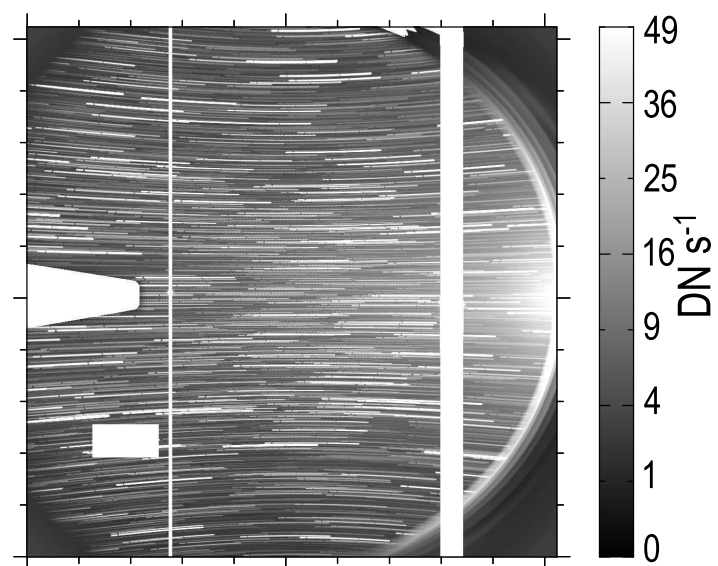

Fig. 2.- Faintest- and brightest-value images from the STEREO/HI-2A Level 1 data spanning 2008 Dec 11-21. (Left) Faintest-value image shows F corona and stray light; (Right) brightest-value image shows moving starfield, spikes from near-spacecraft dust or cosmic rays, and marked data dropouts. The faint image was further processed to reduce starfield, and kept as the F corona model for the data set. Both images have been scaled to 0-50 normalized HI-2 DN $s^{-1}$ and square root scaled to display faint features.

min value, unsharp masked

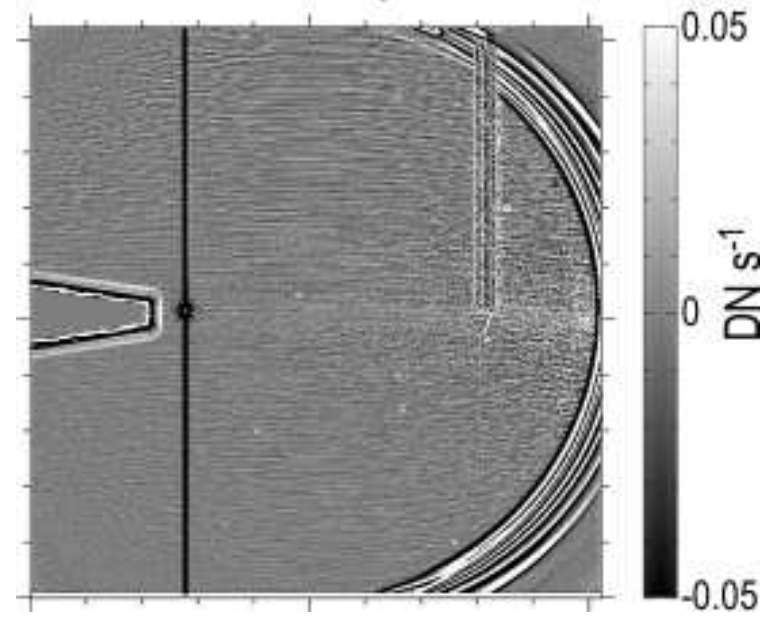

Fig. 3.- Minimum value image from Fig. 2 unsharp masked with a $9 \times 9$ pixel boxcar to show detail. Residual streaks from the starfield are visible throughout, as are artifacts from planetary overexposure and speckles from statistical variation in the F corona. 
random variables in each pixel, and simply ignoring the problem, is skewed median filtering of the background. Selecting the 30th percentile value from each $5 \times 5$ pixel neighborhood removes much of the local brightness from the faint starfield at the cost of increasing F coronal leakage slightly from the skewed noise sample. Figure 4 shows the quality of the fit: the nonstellar fixed pattern is reduced by a factor of approximately 300 by background subtraction with the skewed-median background image. As with other broadband unpolarized Thomson scattering data (e.g. from LASCO C3 Morrill et al. 2006) it is not possible to separate the F coronal (zodiacal light) signal from the fixed portion of the K coronal (Thomson scattered) signal. The subtracted image also demonstrates why there is stellar structure in the minimum image. At the faint end of the stellar brightness distribution, there are stars in most pixels with only isolated darker ones.

\subsection{Celestial background removal}

Removing the stationary and quasi stationary background components leaves the celestial background of stars, galaxies, and other features that remain fixed on the celestial sphere rather than the image plane. The starfield is the most challenging noise source to remove, because individual stars take the forms of spikes on the image, with high gradients. Subtraction of a model starfield requires deeply sub-pixel alignment on the image. The alignment is made more challenging by the fact that the starfield moves on the image plane between exposures as the spacecraft orbits the Sun, and by the fact that the optics impose a nontrivial projection function on the wide-angle field of view. We generated a model starfield from the data themselves, fitting the distortion function $D$ between images as the composition of a parameterized deprojection function from the image plane to the celestial sphere. This is followed by a time-dependent orbital transformation, and reprojection onto the focal plane:

$$
D_{q_{i}}(x, y, \Delta t)=\left(P_{q_{i}} \circ S_{q_{i}}(\Delta t) \circ P_{q_{i}}^{-1}\right)(x, y)
$$

where $P_{q_{i}}$ is the projection function from celestial coordinates to image coordinates and is invariant under nominal operations (though it includes a rotation to orbital coordinates that could in principle change if the instrument pointing changed from the nominal ecliptic-plane

orientation), $S_{q_{i}}$ is the orbital-shift function in ecliptic coordinates and contains all the time dependence in $D$, and the $q_{i}$ are the fit parameters. As a check on the projection correctness we fitted the orbital speed and spacecraft attitude as part of the parameterization. The fit was accomplished by measuring the in-plane coordinate shift between the starfields in a collection of points from two separate images collected five days apart, and adjusting both $P$ and $S(2.5 d)$ to minimize the RMS error between the modeled and measured shifts. Because $S$ is approximately linear in time (treating the orbit as circular), and $P$ is invariant across 
exposures, this yielded a coordinate transformation capable of transforming the starfield between the focal planes of any two exposures at known times in the same general part of the STEREO orbit.

\subsection{Measuring cross-image distortion}

Fitting the functions in Equation (11) requires measuring $D(x, y)$. We measured the function with a regular grid of tiepoints that were used as the basis of correlative fits between two images taken 5 days apart. In our first attempt, we centroided bright stars (on the irregular grid formed by the stars themselves); but the centroid identifications had positional noise at about the 0.2 pixel RMS level, with occasional outliers (in bright stars) of about 0.5 pixel displacement. We attribute this noise source to subpixel variations in detector sensitivity, coupled to the strong gradients in the stellar images, as described by Jackson et al. (2004). Switching to patch correlation between corresponding patches in the earlier and later images yielded much lower noise, which we attribute to dithering of the subpixel gradients across all the stars in each patch (typically dozens to hundreds of faint stars), thereby beating down this noise source via multiple sampling. A further noise source, which is important even to the correlative fits near the edges of the image, is variation in the instrument PSF across the image field of view. Near the edges of the field of view, stars become visible elongated and the correlative fits appear correspondingly noisier. PSF effects are not important near the center of the image plane.

We arrived at a square correlated patch of $31 \times 31$ pixels in size, large enough to yield a large central correlation spike at the proper alignment and null the correlation signal for spurious alignments of different bright stars between the two images. Typical patches contained dozens of individually resolved stars in addition to the unresolved faint starfield. To better equalize the correlation contribution between bright and faint stars, we took the square root of each image patch. Typical correlation coefficients with 1 pixel offset were $0.4-$ 0.6, though a few coincidental missed fits yielded local maximum correlation coefficients as high as 0.80 for misaligned starfields. For the proper subpixel displacement typical correlation coefficients were above 0.995 . The patches were chosen on a regular grid, and missed fits were rejected. In general, fits near the edge of the field of view were missed, along with fits that included a planetary saturation spike in one or the other image.

The initial guess offset, which we used to find the corresponding patch on the later image and seed the correlation fit, was formed based on a horizontal offset of 72 pixels in 5 days. We calculated the correlation coefficient between the two images 5 days apart on a $15 \times 15$ grid of integer pixel offsets around the horizontal offset location, and the subpixel fit was seeded with 
the best offset among the trial offsets. Further alignment was fit using a simplex/amoeba fitter (Press et al. 1989) with initial simplex radius of 0.75 pixels, centered on the best trial alignment. Patches were rejected if either the original or the shifted patch, with margins, intersected the edge of the telescope image plane or contained even one saturated pixel, or if the final best-fit correlation coefficient between the patches in the earlier and later frame was below 0.99. Figure 5 shows the horizontal and vertical components of the measured $D(x, y, 2.5 d)$, after removal of the base horizontal 72-pixel offset.

The $D_{q i}$ distortion function was assembled from parameterized components as in Equation (11). The amount of displacement in $S$ was a parameter of the fit, to verify that the spacecraft's orbital speed was reproduced by the fitter. The $P_{q i}$ was a perspective projection of the celestial sphere with variable camera angle and $\mu$ parameter (distance from projecting point to the center of the sphere), as described by Snyder (1987) and Brown et al. (2009). We hoped to use the starfield, as did Brown et al., but to characterize the frame-to-frame offsets to better than their reported 1 pixel precision by refining the family of coordinate transformations used to relate celestial and STEREO focal-plane coordinates.

The initial guess $P$ was a stereographic projection of the celestial sphere (i.e. $\mu=1)$ and the initial guess $S$ was based on a $1^{\circ} \mathrm{d}^{-1}$ rotation about an axis parallel to the vertical axis of the image. The $q_{i}$ were iterated through an amoeba fitting process to find the best overall $D$ using the sum-of-squares distance between the two sets of tiepoint pixel coordinates after transformation to the central time between the two images.

Initial free parameters were: pointing of the projection center relative to image center (X and Y), roll and "B" (out-of-plane) angle of the projective plane relative to the ecliptic pole, orbital rotation $\theta$, and projection distortion parameter $\mu$. The roll and $\mathrm{B}$ angles, in particular, were treated as instrumental constants across the dataset. This is a valid simplification for a single few-day data set, though for a more general pipeline process the two angles should be allowed to change according to spacecraft orbital phase relative to the celestial nodes of the orbit.

The initial fitting process led to a 6-parameter coordinate transformation with a residual RMS error of 0.35 pixel between the measured and modeled distortion parameters at the kept tiepoints, compared to the initial error (depicted in Figure 5) of 3.6 pixels. An order of magnitude improvement isn't bad, but more improvement was possible. The residuals contained obvious errors in a quadrant pattern for vertical displacement and in a vertical striation pattern for horizontal displacement, and each was treated with ad-hoc perturbation functions of known pattern and variable amplitude, adding two more parameters to the fit. 
The horizontal displacement ad hoc distortion was

$$
x^{\prime}=x+\beta \sin \left(\frac{3 \pi}{2} \frac{(x-512)}{512}\right) \cos \left(\frac{\pi}{2} \frac{(y-512)}{512}\right)
$$

and the vertical displacement ad hoc distortion was

$$
y^{\prime}=y+\alpha \sin ^{2}\left(\frac{2 \pi}{3} \frac{(x-512)}{512}\right) \sin \left(\frac{\pi}{2} \frac{(y-512)}{512}\right)
$$

where $\alpha$ and $\beta$ are the fitted amplitude coefficients and $x$ and $y$ are pixel index coordinates in the original image plane before any other coordinate transforms were applied, i.e.

$$
P_{\text {final }}^{-1}=P_{6-\text { param }}^{-1} \circ f_{\text {adhoc }}(x, y) .
$$

With the $\alpha$ and $\beta$ parameters, a total of eight fit parameters were fitted to the measured displacements of each patch. The ad hoc functions had the correct performance for the central region of the image plane, but diverged slightly in the periphery of the valid images. For the purpose of this exploratory study, we simply discarded the region of poorer convergence, limiting the study to a $400 \times 750$ pixel region at the center of the image plane. Limiting the field of view is a good choice as it limits other edge effects such as vignetting and PSF variation at the periphery of the image plane, and also allows us to avoid the two top-tobottom saturated regions in this data set (one from Earth and one from Venus).

After iterating the distortion fit with the two ad-hoc correction functions, the distortion residual error was 0.09 pixel RMS in the target region. The residuals are shown in Figure 6. Although this represents a large improvement in characterization of the starfield motion, with better or more complete selection of final correction functions there is still room for further improvement, as the residuals still contain visually identifiable patterns.

The advantage of fitting the pixel displacement function as in Equation (11) is that new displacements can be computed using only the time difference between any two images in the same data set. We used the fitted $P(x, y)$ and a computed $S(\Delta t)$ to warp every HI-2A image from 11 Dec 2008 at 00:09:21 through 21 Dec 2008 at 22:09:21 to a central time of 14 Dec 2008 at 00:09:21, to fix the starfield in the datacube. That fixed starfield was then used to calculate the median value from every pixel, which formed a background starfield image that could be subtracted from the cube. Because of the high gradients around the bright stars, the median subtracted images still contained paired stellar images due to the subpixel misalignments. These took the form of dual (positive-going and negative-going) spikes around each bright star on opposite sides of the PSF core (2-3 pixels apart) and, near the periphery of the image where the PSF variation becomes important, triple spikes (two positive-going and one negative-going, or vice versa) around each bright star due to the 
changing width of the PSF. We eliminated these artifacts using a $7 \times 7$ pixel running median filter - each pixel was replaced by the median of the values in a $7 \times 7$ square around itself. The $7 \times 7$ median filter effectively blurs the images by $0.5^{\circ}$, well within the expected motion blur from $300 \mathrm{~km} / \mathrm{s}$ solar wind, which is of order $2^{\circ}$ at $0.5 \mathrm{AU}$ distance from the observer. The median-filtered images were then given similar treatment to the $\mathrm{F}$ corona subtraction step above. We identified the distribution of brightness values in each pixel, selected the 10th percentile as a background value, and subtracted it from each distorted image. The results of these steps are given in Figure 7.

After starfield removal, the remaining visual features have an amplitude of approximately $0.1 \mathrm{DN}^{-1}$. Near the center of the data set, the starfield null is good and essentially no structured stellar artifacts are seen. Nearer the extremities in either space or time, the starfield begins to "punch through" due to residual errors in the fitting function and to PSF variation across the original image field. However, the background level is no longer dominated by stars but by second-order effects due to the motion of the F corona during the data collection period.

Even near the center of the image, the starfield remains faintly visible in the processed data as randomly fluctuating light/dark spots at the location of the pre-subtraction star. The fluctuations have no discernible (to us) spatial or temporal signature that could be exploited to reduce this noise source further, and they do not have the characteristic twoor three-peak structure characteristic of misaligned stars. We attribute the fluctuations to slight variations in the effective gain of the detector depending on the phase of the stellar image relative to the pixel grid as described by Jackson et al. (2004). This is the same effect that forced us to use patch correlation rather than direct centroiding of stars to measure the distortion function.

Throughout the fitting and analysis process, careful attention to resampling is critically important because starfields stress the resampling operation and the usual interpolative shortcuts will spoil the data. When the starfield is "frozen" by resampling with bilinear interpolation (as is common in the heliophysics imaging community), small ripples are visible in the resulting starfield movie, corresponding to small motions of the stellar centroids of up to 0.5 pixel in the resampled data and peak amplitude fluctuations of tens of percent. In that case the starfield nulling operation is completely spoiled. To eliminate this type of artifact, we used spatially variable Jacobian-optimized resampling as described by DeForest (2004).

As an interesting aside, small, faint objects are easily visible in movies of the frozen starfield. When visually inspecting the frozen-starfield movie, we noticed a small moving object that proved to be Uranus (Figure 8). 


\subsection{Residual F corona removal}

After removal of the bulk starfield and fixed $\mathrm{F}$ corona, we have reduced the dynamic range of the artifacts from $\sim 30 \mathrm{DN} \mathrm{s}^{-1}$ to $\sim 0.1 \mathrm{DN} \mathrm{s}^{-1}$, a factor of 300 improvemnt. At this level, second order effects in the $\mathrm{F}$ corona come into play, seen as the large scale variation in brightness in Figure 7. The residual brightness enters because the $\mathrm{F}$ corona is not completely time-independent as assumed in $\$ 3.1$. We removed the brightness variation by making a 3rd order fit to the time varying brightness of each pixel across the full 11 day observing window, and subtracting that 3rd order fit from the brightness of each pixel. The automated fit didn't necessarily find the correct offset to preserve the zero point, so after subtracting the 3rd order fit, we subtracted from each pixel the minimum value of the $5 \times 5$ pixel median centered on that pixel, over the whole 12 day run. This process yielded relatively clean images of the $\mathrm{K}$ coronal component of the images, but some stellar artifacts remained visible. In particular, although the spatial median filter in $\$ 3.2$ removed the large spikes due to stellar images, stars are preserved by two effects:

1. the spikes offset the distribution of values in the remaining pixels in each $5 \times 5$ pixel region, so that the median does change slightly when the distribution of values includes a spike (though not as much as does the mean);

2. the instrument point-spread function is not perfect, so that around each bright star is a small halo of points that are brighter than deep space by a value comparable to the signal for which we are looking.

Both effects give rise to bright artifacts that are stretched in the direction of stellar motion on the detector, because they influence the signal in the fixed-focal-plane segment in \$3.1, where the stars are in motion. These halos are particularly visible in Figure 7 as horizontal bright artifacts. They are difficult to remove with single-image or pairwise image processing, because they have approximately the same signal strength and size as features of interest.

\subsection{Fourier moving-object filter}

We removed the remaining celestial-frame fixed artifacts with a Fourier filtering process. The entire 12 day image set was Fourier transformed in $3-\mathrm{D}$ to $k_{x}, k_{y}, \omega$ space, and filtered with a moving-object filter that rejected all signal associated with wave speed slower than two image pixels per frame and all fixed signal in the current (celestial) coordinate system. The orbital motion of the starfield in the FOV is approximately 1.2 pixels per frame. For HI-2A, this motion is in the opposite direction to the solar wind propagation in the field of 
view, so our two pixels per frame limit corresponds to less than one pixel per frame in the original data.

To perform the filtering, we kept all Fourier energy inside in a cone with slope of 2 pixels/frame near the $\omega$ axis in the celestial-frame resampled data, and rejected Fourier energy outside the cone. To prevent ringing, we applied a Hanning-style cosine rolloff with width $1 / 3$ of the base filter width, centered on the cutoff frequency. This process makes use of the fact that the solar wind moves in the field of view, while the artifacts are either fixed or slow-moving, and has been used in a solar context primarily in the opposite sense - to remove $\mathrm{P}$ mode oscillations from solar surface magnetogram sequences (Hagenaar \& Shine 2005; Lamb et al. 2008). Fast moving objects in magnetogram sequences are typically $\mathrm{P}$ modes that have high phase speed, and are rejected, while slow moving objects are the desired signal. Here, the sense is reversed, and we preserve moving solar wind features while rejecting the fixed pattern of the starfield.

In addition to slow moving components, we rejected the fixed $(\omega=0)$ component of the Fourier image, which eliminates both the mean brightness and pattern background. This loss was repaired post facto by identifying the "zero point" of each pixel to be the minimum value of a $25 \times 25 \times 5$ voxel average centered on each voxel in the $3-\mathrm{D}(x, y, t)$ data space. When the zero point is subtracted, the remaining signal contains only the brightness component contained in moving features. The result is demonstrated in Figure 9, which shows the same three frames (out of 121) as in Figure 7, after full processing in celestial coordinates.

\subsection{Conversion to focal plane coordinates}

After processing, we resampled the filtered images back into focal plane coordinates to yield a dataset in the original HI-2 coordinate system, with the background removed. We also applied the preliminary HI-2 calibration of $1.1 \times 10^{-14} \mathrm{~B}_{\odot} \mathrm{DN}^{-1} \mathrm{~s}(\mathrm{C}$. Eyles, priv. comm.). The final result is shown in Figure 10 and, in the digital edition of this article, in the attached movie file.

\section{Results}

Figure 10 shows extracted still frames from the data set and, in the digital edition of this article, a movie of the entire data set in original observing coordinates. The images

are cropped to avoid both Venus and the Earth, and therefore show only a section of the HI-2 field of view, spanning about $25^{\circ} \times 60^{\circ}$. The FOV moves gradually to the right as time 
increases, because the cropping, starfield processing, and Fourier filtering were carried out in celestial coordinates before being transformed back into observing coordinates.

The brightness scale of Figure 10 is $0-2.5 \times 10^{-16} \mathrm{~B}_{\odot}$; individual features can be observed at brightnesses of $\sim 2 \times 10^{-17} \mathrm{~B}_{\odot}$. A great deal of structure appears in the sequence, and we have identified a number of features that can be isolated from the background solar wind activity. The level of variance in the solar wind is itself remarkable.

HI-2 observations of specific classes of moving features (Sheeley et al. 2008a) have revealed variability in this altitude range, but to our knowledge this is the first algorithm capable of fixed computed-background subtraction to reduce image contamination below the brightness range of fine-scale features in the solar wind and enabling a direct view of evolving structures as they propagate. More detailed scientific analysis will appear in a following paper; here, we confine ourselves to a first-look examination of the data.

The CME itself first appears in the image dated 2008-12-15 at 00:09:21 in Figure 10 but a number of distinct features in the solar wind can be identified and tracked prior to its appearance. They appear across the top row and we have labeled them "Solar wind puffs". The second and third row shows the passage of the CME and a number of accompanying features that can also be identified and tracked, including "remnant loops" that may be indicative of flux rope structure and that surround an empty cavity or void. The structure slightly ahead of the CME in the second and third frames of the second row (2008-1215T06:09:21 and T12:09:21) may be a CME forerunner (Jackson \& Hildner 1978) and the bright structure toward the south of the CME across the third row may be a prominence or streamer blowout. We have also identified features flowing in the wake of the CME, two different types have been labeled "Plasmoid at CME cusp" and "CME trailing cusp" in the bottom two rows. The former is aligned near the center of the CME and appears to be either a related sympathetic eruption or part of the original magnetic structure comprising the pre-launch coronal field. The latter has a concave-outward V shape and straddles the solar equatorial region, meaning that it may be associated with the heliospheric current sheet.

Behind the CME front in Figure 10 are two clear, dark voids. Voids behind CMEs have been detected remotely via radio interplanetary scintillation (Tappin et al. 1983; Tappin 1987; Tappin \& Howard 2010) but have not been directly imaged before at such high elongations, because heliospheric imaging studies have either used running difference images, thereby confusing dark regions with bright regions that were present in the previous image, or have not achieved the level of sensitivity required to observe the voids and trailing faint structure. We tentatively identify the voids with the cavity in the the classical three-part CME often observed in coronagraphs (Sime et al. 1984) and also with the region of reduced 
density often observed accompanying (and sometimes identified as a signature of) in-situ magnetic clouds (e.g. Wu \& Lepping 2002; Wei et al. 2003). When the feature impacted the Wind spacecraft a void was detected in-situ (see below).

Figure [11 shows the data sequence in the form of an elongation-time "J-plot", produced by selecting a position angle slice across an elongation-time-intensity data cube, and utilized widely by the HI community (e.g. Davies et al. 2009). A number of position angle slices have been chosen, measured relative to the ecliptic plane. Many of the features are labeled where they correspond with those in the movie frames.

Figure 12 shows a J-plot for the ecliptic plane $\left(+5^{\circ}\right.$ relative to STEREO-A heliographic coordinates during this time of year) built from the processed data sequence. The Earth is indicated as a dashed horizontal line near $70^{\circ}$ elongation. This allows direct comparison between the HI-2 features and in-situ density data, and the density measured by Wind/SWE for the same time period is also shown above. Most of the features that we can track in the HI-2 dataset at this heliospheric latitude also appear in the in-situ data. Several labeled features from Figure 10 are indicated in Figure 12 and extended to the Earth with a dashed line. A few features (e.g. the second puff) do not appear or cannot be traced all the way to Earth (e.g. P4), either because they miss the Earth's heliospheric longitude are weak against the background or other brighter features. Three-dimensional analysis is planned using combined background-subtracted data from both of the STEREO spacecraft and from other auxiliary data sets.

\section{Discussion}

We have demonstrated extraction of a quantitative solar wind imaging signal from a HI2 image sequence containing 3-4 orders of magnitude brighter background than the desired data. We are able to approach, but not achieve, the photon counting noise limit in the STEREO/HI-2 instrument.

The limiting factors in the final image quality of our data set are (1) stellar background "print-through", which we attribute primarily to subpixel variations in CCD sensitivity coupled to the relative phase of the pixel grid and the star's location; and (2) motion blur from the standard two hour accumulation time for HI-2, with corresponding blur of up to a few degrees with typical feature speed ranges (e.g. Howard \& Simnett 2008). The stellar background print-through dominates over photon counting noise in the feature size range that is observable in our data set, by a factor of about 3 .

Because we use the image stream itself as a background source, exploiting differences 
in behavior and spatial distribution to distinguish desired data from the background, it is not possible to extract absolute brightnesses - only absolute "excess brightnesses" of moving features, as is typical for other unpolarized Thomson-scattering detectors such as SOHO/LASCO (Brueckner et al. 1995). Further, the final filtering step makes use of the fact that the solar wind apparent speed is fast compared to the angular orbital speed of the spacecraft, and therefore the processd data are not suited to imaging of features in the solar wind with little apparent motion, such as corotating interaction regions.

Further improvement of this type of data would require (1) shorter effective exposure times or better motion compensation; (2) a way to minimize stellar print-through; and (3) an absolute measurement of the celestial background independent of the desired Thomsonscattered signal. Happily, all three are technically feasible: (1) may be accomplished with a larger instrument or with higher downlinked data volume; (2) may be accomplished with higher data volume by either defocusing the optics or dithering the exposure on the CCD between individual camera exposured during image accumulation; and (3) polarization can be used to separate the Thomson scattered signal from the background signal. Polarization, in particular, is important because the stars and galaxy, which are far more challenging than the F corona to remove, are unpolarized in broadband visible light, and the slight $\mathrm{F}$ coronal polarization follows established models of particulate scattering (Leinert et al. 1997).

We are able to reveal absolute excess brightness of solar wind and CME structures with a $\mathrm{S} / \mathrm{N}$ ratio of up to 30 in $1.5^{\circ}$ patches of image, and demonstrate the presence of "voids" and complex structure in the wind stream more than $60^{\circ}$ (or $\sim 0.85 \mathrm{AU}$ ) from the Sun. Many features that are visible, including "V" shaped structures surrounding the heliospheric current sheet near the plane of the ecliptic, are to be expected from the wind speed gradient but have not been observed directly this far from the Sun.

While the presence of structure in the solar wind has been known since the first in-situ measurements of particle density, and some wind transients have been detected with the STEREO/HI-2 imagers, to our knowledge this is the first report of quantitative white light imaging of detailed solar wind structure so far from the Sun. That is not surprising given the challenges inherent in separating the bright starfield from the faint Thomson signal, which requires both deep subpixel understanding of the instrument's projection function and careful attention to the mechanics of data resampling. We were aided in both of these goals by extensive use of Perl Data Language (Glazebrook \& Economou 1997), a scientific computing package that includes a framework for parameterizing and combining coordinate transformations, and for optimized data resampling (DeForest 2004).

Efforts currently in progress, to be followed up in future reports, include three-dimensional reconstruction of feature complexes using dual spacecraft views and image analysis (e.g. 
Howard 2011), direct association of CME structure at high elongation with corresponding structure at the Sun via continuous tracking from launch to $1 \mathrm{AU}$; and a more detailed analysis of the small scale features revealed in the reprocessed data.

The analysis technique we present here is based on batch processing of images and is limited to a few days per batch, partly by practical constraints of computer RAM and partly by motion of the starfield (which induces a trade-off between length of run and field of view); however, an open-ended processing pipeline has been prototyped, and works by merging the results of multiple batch-processed data sets. That technique and its results will be presented in an article to follow this one.

The authors thank the HI team for the kind use of their calibrated data. STEREO/HI was developed by a collaboration including the Rutherford Appleton Laboratory and the University of Birmingham (UK), the Centre Spatial de Liége (Belgium), and the Naval Research Laboratory (US). The work for the present study was supported in part by the NSF/SHINE Competition, Award 0849916, and in part by the NASA Heliophysics program through grant NNX10AC05G. SJT is supported at NSO by the USAF under a Memorandum of Agreement. The data reduction utilized the free Perl Data Language, which is available

at "http://pdl.perl.org', The authors also gratefully acknowledge insightful discussions with S. Antiochos, S. Crothers, C. Eyles, N. Schwadron, and N. Sheeley.

\section{REFERENCES}

Bewsher, D., Brown, D. S., Eyles, C. J., Kellett, B. J., White, G. J., \& Swinyard, B., 2010, Solar Phys., 264, 433.

Brown, D. S., Bewsher, D., \& Eyles, C. J. 2009, Solar Phys., 254, 185.

Brueckner, G. E., Howard, R. A., Koomen, M. J., Korendyke, C. M., Michels, D. J., Moses, J. D., Socker, D. G., Dere, K. P., Lamy, P. L., Llebaria, A., Bout, M. V., Schwenn, R., Simnett, G. M., Bedford, D. K., \& Eyles, C. J., 1995, Solar Phys., 162, 357.

Clover, J. M., Jackson, B. V., Buffington, A., Hick, P. P., \& Bisi, M. M., 2010, Astrophys. J., $713,394$.

Davies, J. A., Harrison, R. A., Rouillard, A. P., Sheeley, N. R., Jr., Perry, C. H., Bewsher, D., Davis, C. J., Eyles, C. J., Crothers, S. R., \& Brown, D. S., 2009, Geophys. Res. Lett., 36, L02102, doi:10.1029/2008GL036182 
Davis, C. J., Davies, J. A., Lockwood, M., Rouillard, A. P., Eyles, C. J., \& Harrison, R. A., 2008, Geophys. Res. Lett., 36, L08102, doi:10.1029/2009GL038021.

DeForest, C. E., 2004, Solar Phys., 219, 3.

DeForest, C. E., Hagenaar, H. J., Lamb, D. A., Parnell, C. E., \& Welsch, B. T., 2007, Astrophys. J., 666, 576.

Eyles, C. J., Simnett, G. M., Cooke, M. P., Jackson, B. V., Buffington, A., Hick, P. P., Waltham, N. R., King, J. M., Anderson, P. A., \& Holladay, P. E., 2003, Solar Phys., 217,319

Eyles, C. J., Harrison, R. A., Davis, C. J., Waltham, N. R., Shaughnessy, B. M., MapsonMenard, H. C. A., Bewsher, D., Crothers, S. R., Davies, J. A., Simnett, G. M., Howard, R. A., Moses, J. D., Newmark, J. S., Socker, D. G., Halain, J.-P., Defise, J.-M., Mazy, E., \& Rochus, P., 2009, Solar Phys., 254, 387.

Glazebrook, K. \& Economou, F., 1997, The Perl Journal, 5, 5.

Hagenaar, H. J., \& Shine, R. S., 2005, Proc. 11th European Solar Phys. Meet. (ESA SP-600), Leuven, Belgium, Danesy, D., Poedts, S., De Groof, A, \& Andries, J (eds.), p.60.1.

Harrison, R. A., Davis, C. J., Eyles, C. J., Bewsher, D., Crothers, S. R., Davies, J. A., Howard, R. A., Moses, D. J., Socker, D. G., Newmark, J. S., Halain, J.-P., Defise, J.-M., Mazy, E., Rochus, P., Webb, D. F., \& Simnett, G. M., 2008, Solar Phys., 247, 171.

Harrison, R. A., Davies, J. A., Rouillard, A. P., Davis, C. J., Eyles, C. J., Bewsher, D., Crothers, S. R., Howard, R. A., Sheeley, N. R., Jr., Vourlidas, A., Webb, D. F., Brown, D. S., \& Dorrian, G. D., 2009, Solar Phys., 256, 219.

Howard, T. A., J. Atmos. Solar Terr. Phys., 2011, in press, doi:10.1016/j.jastp.2010.08.009.

Howard, R. A., Moses, J. D., Vourlidas, A., Newmark, J. S., Socker, D. G., Plunkett, S. P., Korendyke, C. M., Cook, J. W., Hurley, A., Davila, J. M., Thompson, W. T., St Cyr, O. C., Mentzell, E., Mehalick, K., Lemen, J. R., Wuelser, J. P., Duncan, D. W., Tarbell, T. D., Wolfson, C. J., Moore, A., Harrison, R. A., Waltham, N. R., Lang, J., Davis, C. J., Eyles, C. J., Mapson-Menard, H., Simnett, G. M., Halain, J. P., Defise, J. M., Mazy, E., Rochus, P., Mercier, R., Ravet, M. F., Delmotte, F., Auchere, F., Delaboudiniere, J.-P., Bothmer, V., Deutsch, W., Wang, D., Rich, N., Cooper, S., Stephens, V., Maahs, G., Baugh, R., McMullin, D., \& Carter, T., 2008, Space Sci. Rev., 136, 67. 
Howard, T. A., \& Simnett, G. M., 2008, J. Geophys. Res., 113, A08102, doi:10.1029/2007JA012920.

Howard, T. A., Webb, D. F., Tappin, S. J., Mizuno, D. R., \& Johnston, J. C., 2006, J. Geophys. Res., 111, A04105, doi:10.1029/2005JA011349.

Jackson, B. V., Buffington, A., Hick, P. P., Altrock, R. C., Figueroa, S., Holladay, P. E., Johnston, J. C., Kahler, S. W., Mozer, J. B., Price, S., Radick, R. R., Sagalyn, R., Sinclair, D., Simnett, G. M., Eyles, C. J., Cooke, M. P., Tappin, S. J., Kuchar, T., Mizuno, D., Webb, D. F., Anderson, P. A., Keil, S. L., Gold, R. E., \& Waltham, N. R., 2004, Solar Phys., 225, 177.

Jackson, B. V., \& Hildner, E., 1978, Solar Phys. 60, 155, 1978.

Jackson, B. V., Howard, R. A., Sheeley, N. R., Jr., Michels, D. J., Koomen, M. J., \& Illing, R. M. E., 1985, J. Geophys. Res., 90, 5075.

Kaiser, M. L., Kucera, T. A., Davila, J. M., St. Cyr, O. C., Guhathakurta, M. \& Christian, E., 2008, Space Sci. Rev., 136, 5.

Lamb, D. A., DeForest, C. E., Hagenaar, H. J., Parnell, C. E., \& Welsch, B. T., 2008, Astrophys. J., 674, 520.

Leinert, C., Bowyer, S., Haikala, L. K., Hanner, M. S., Hauser, M. G., Levasseur-Regourd, A.-C., Mann, I., Mattila, K., Reach, W. T., Schlosser, W., Staude, H. J., Toller, G. N., Weiland, J. L., Weinberg, J. L. \& Witt, A. N., 1997, Astron. Astrophys. Suppl. $127,1$.

Liu, Y., Davies, J. A., Luhmann, J. G., Vourlidas, A., Bale, S. D., \& Lin, R. P., 2010, Astrophys. J., 710, L82.

Lugaz, N., Hernandez-Charpak, J. N., Roussev, I. I., Davis, C. J., Vourlidas, A., \& Davies, J. A., 2010, Astrophys. J., 715, 493.

Morrill, J. S., Korendyke, C. M., Brueckner, G. E., Giovane, F., Howard, R. A., Koomen, M., Moses, D., Plunkett, S. P., \& Vourlidas, A., 2006, Solar Phys., 233, 331.

Möstl, C., Temmer, M., Rollett, T., Farrugia, C. J., Liu, Y., Veronig, A. M., Leitner, M., Galvin, A. B. \& Biernat, H. K., 2010, Geophys. Res. Lett., 37, doi:10.1029/2010GL045175. 
Phillips, J. L., Bame, S. J., Feldman, W. C., Goldstein, B. E., Gosling, J. T., Hammond, C. M., McComas, D. J., Neugebauer, M., Scime, E. E., \& Suess, S. T., 1995, Science, 268,1030 .

Press, H. P., et al., 1989: "Numerical Recipes" (Cambridge University Press).

Richter, I., Leinert, C., \& Planck, B., 1982, Astron. Astrophys., 110, 115.

Rickett, B. J., \& Coles, W. A., 1991, J. Geophys. Res., 96, 1717.

Rouillard, A. P., Davies, J. A., Forsyth, R. J., Rees, A., Davis, C. J., Harrison, R. A., Lockwood, M., Bewsher, D., Crothers, S. R., Eyles, C. J., Hapgood, M., \& Perry, C. H., 2008, Geophys. Res. Lett., 35, L10110, doi:10.1029/2008GL033767.

Rouillard, A. P., Davies, J. A., Lavraud, B., Forsyth, R. J., Savani, N. P., Bewsher, D., Brown, D. S., Sheeley, N. R., Jr., Davis, C. J., Harrison, R. A., Howard, R. A., Vourlidas, A., Lockwood, M., Crothers, S. R., \& Eyles, C. J., 2010, J. Geophys. Res., 115, A04103, doi:10.1029/2009JA014471.

Schwenn, R., 1990, in Physics of the Inner Heliosphere Vol. 1, Large-Scale Phenomena (Springer, Berlin), 99.

Sheeley, N. R., Walters, J. H., Wang, Y.-M., \& Howard, R. A., 1999, J. Geophys. Res., 104, 24739 .

Sheeley, N. R., Jr., Wang, Y.-M., Hawley, S. H., Brueckner, G. E., Dere, K. P., Howard, R. A., Koomen, M. J., Korendyke, C. M., Michels, D. J., Paswaters, S. E., Socker, D. G., St. Cyr, O. C., Wang, D., Lamy, P. L., Llebaria, A., Schwenn, R., Simnett, G. M., Plunkett, S., \& Biesecker, D. A., 1997, Astrophys. J., 484, 472.

Sheeley, N. R., Jr., Herbst, A. D., Palatchi, C. A., Wang, Y.-M., Howard, R. A., Moses, J. D., Vourlidas, A., Newmark, J. S., Socker, D. G., Plunkett, S. P., Korendyke, C. M., Burlaga, L. F., Davila, J. M., Thompson, W. T., St Cyr, O. C., Harrison, R. A., Davis, C. J., Eyles, C. J., Halain, J.-P., Wang, D., Rich, N. B., Battams, K., Esfandiari, E., \& Stenborg, G., 2008a, Astrophys. J., 674, L109.

Sheeley, N. R., Jr., Herbst, A. D., Palatchi, C. A., Wang, Y.-M., Howard, R. A., Moses, J. D., Vourlidas, A., Newmark, J. S., Socker, D. G., Plunkett, S. P., Korendyke, C. M., Burlaga, L. F., Davila, J. M., Thompson, W. T., St Cyr, O. C., Harrison, R. A., Davis, C. J., Eyles, C. J., Halain, J.-P., Wang, D., Rich, N. B., Battams, K., Esfandiari, E., \& Stenborg, G., 2008b, Astrophys. J., 675, 853. 
Sheeley, N. R., Lee, D. D.-H., Casto, K. P., Wang, Y.-M., \& Rich, N. B., 2009, Astrophys. J., 694, 1471.

Sheeley, N. R., Jr., \& Rouillard, A. P., 2010, Astrophys. J., 715, 300.

Sime, D. G., MacQueen, R. M., \& Hundhausen, A. J., 1984, J. Geophys. Res., 89, 2113.

Snyder, J. P., 1987: "Map Projections", USGS Professional Paper 1395, US Govt. Printing Office (Washington).

Tappin, S. J., Hewish, A, \& Gapper, G. R., 1987, Plan. \& Sp. Sci., 31, 1171.

Tappin, S. J., 1987, Plan. \& Sp. Sci., 35, 271.

Tappin, S. J., Buffington, A., Cooke, M. P., Eyles, C. J., Hick, P. P., Holladay, P. E., Jackson, B. V., Johnston, J. C., Kuchar, T., Mizuno, D., Mozer, J. B., Price, S., Radick, R. R., Simnett, G. M., Sinclair, D., Waltham, N. R., \& Webb, D. F., 2004, Geophys. Res. Lett., L02802, doi:10.1029/2003GL018766.

Tappin, S. J., \& Howard, T. A., 2009a, Astrophys. J., 702, 862.

Tappin, S. J., \& Howard, T. A., 2009b, Space Sci Rev., 147, 55.

Tappin, S. J. \& Howard, T. A., 2010, 265, 159.

Webb, D. F., Howard, T. A., Fry, C. D., Kuchar, T. A., Odstrcil, D., Jackson, B. V., Bisi, M. M., Harrison, R. A., Morrill, J. S., Howard, R. A., \& Johnston, J. C., 2008, Solar Phys., 256, 239.

Wei, F., Liu, R., Fan, Q., \& Feng, X., 2003, J. Geophys. Res., 108, 1263, doi:10.1029/2002JA009511.

Wu, C.-C., \& Lepping, R.P., 2002, J. Geophys. Res., 107, 1314, doi:10.1029/2001JA000161. 


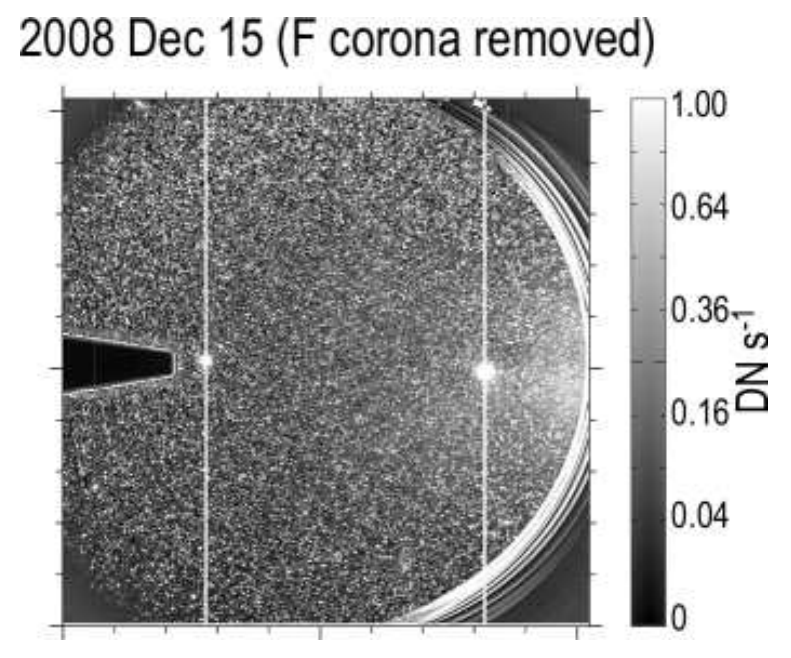

Fig. 4.- Background-subtracted version of Figure 1, using the background derived via the 5th percentile image of an 11 day dataset, with skewed-median smoothing as described in the text. The dynamic range is $25 \times$ more sensitive than Figure 1, The fixed brightness pattern containing the $\mathrm{F}$ corona has been reduced by a factor of approximately 300 but is still faintly visible at right. Many individual stars greatly exceed the dynamic range of this plot. 

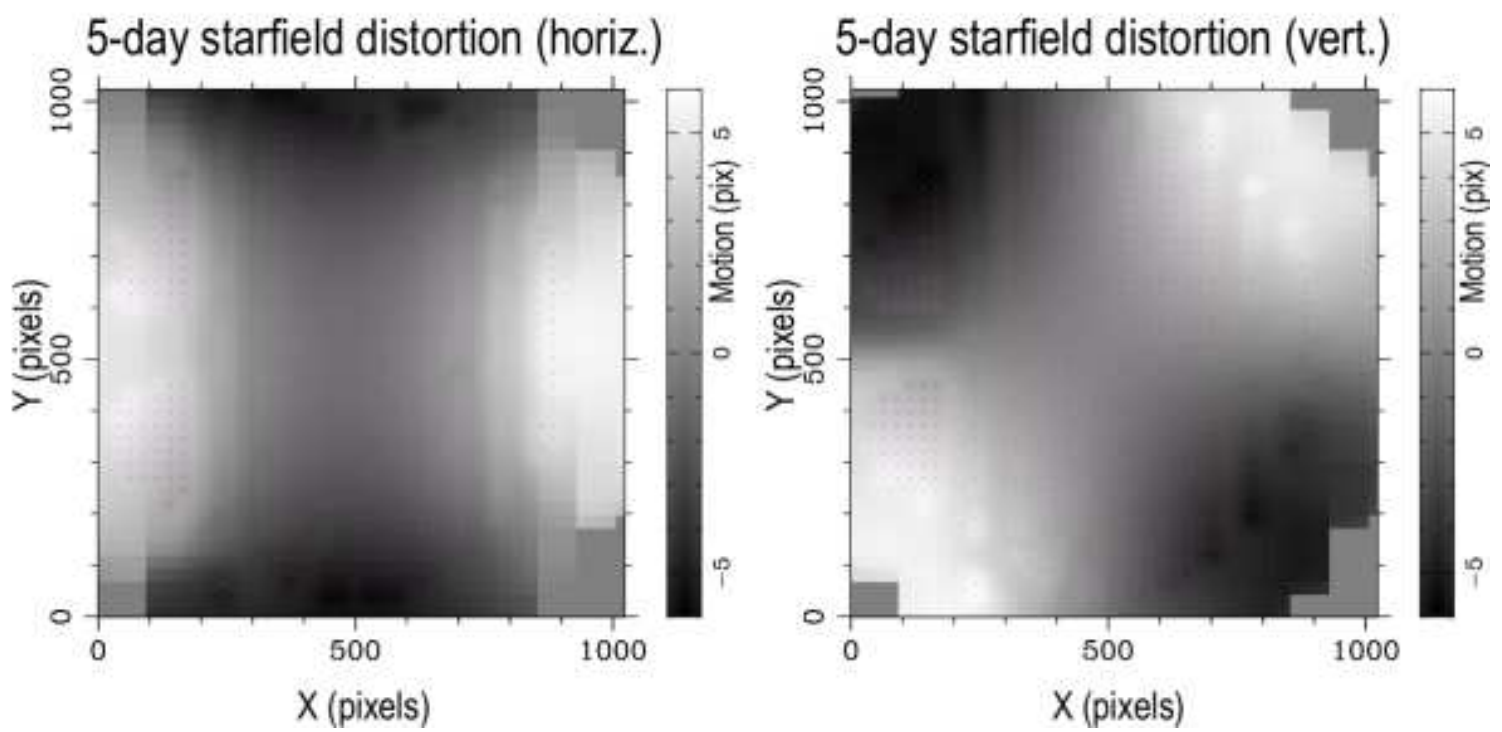

Fig. 5.- Initial measured nonlinear distortion function for STEREO/HI-2A shows measured horizontal and vertical distortions as a function of position over a 5 day interval in Dec 2008 in the HI-2A field of view. Kept correlation patch centers are marked and form the data comparison points for the subsequent fit of the coordinate transformation. For display, the images are interpolated between patch centers using an inverse square distance-weighted average.
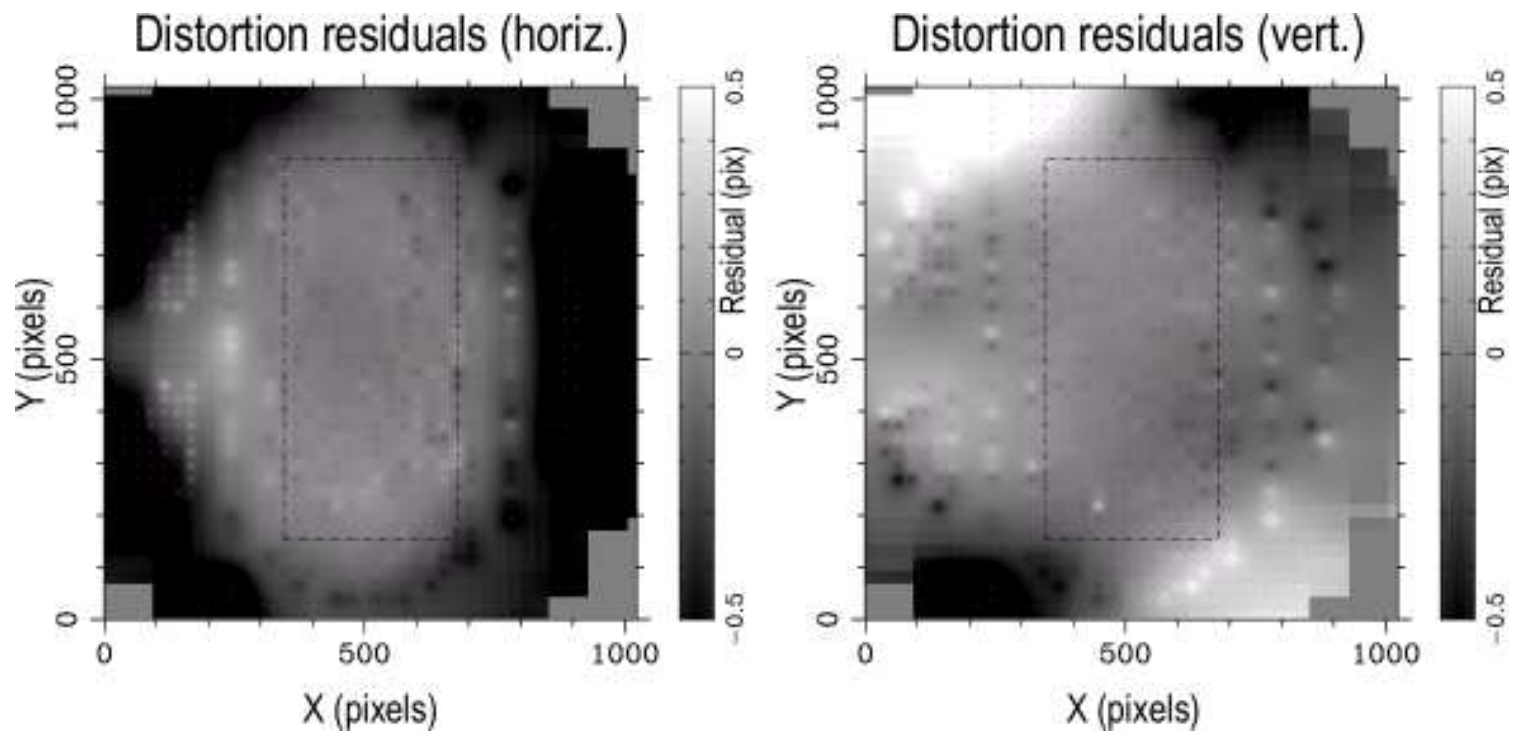

Fig. 6.- Residual distortion error after starfield distortion fitting is 0.09 pixel RMS in the central rectangle (compare to Figure 5). Locations where the distortion was measured are marked. 

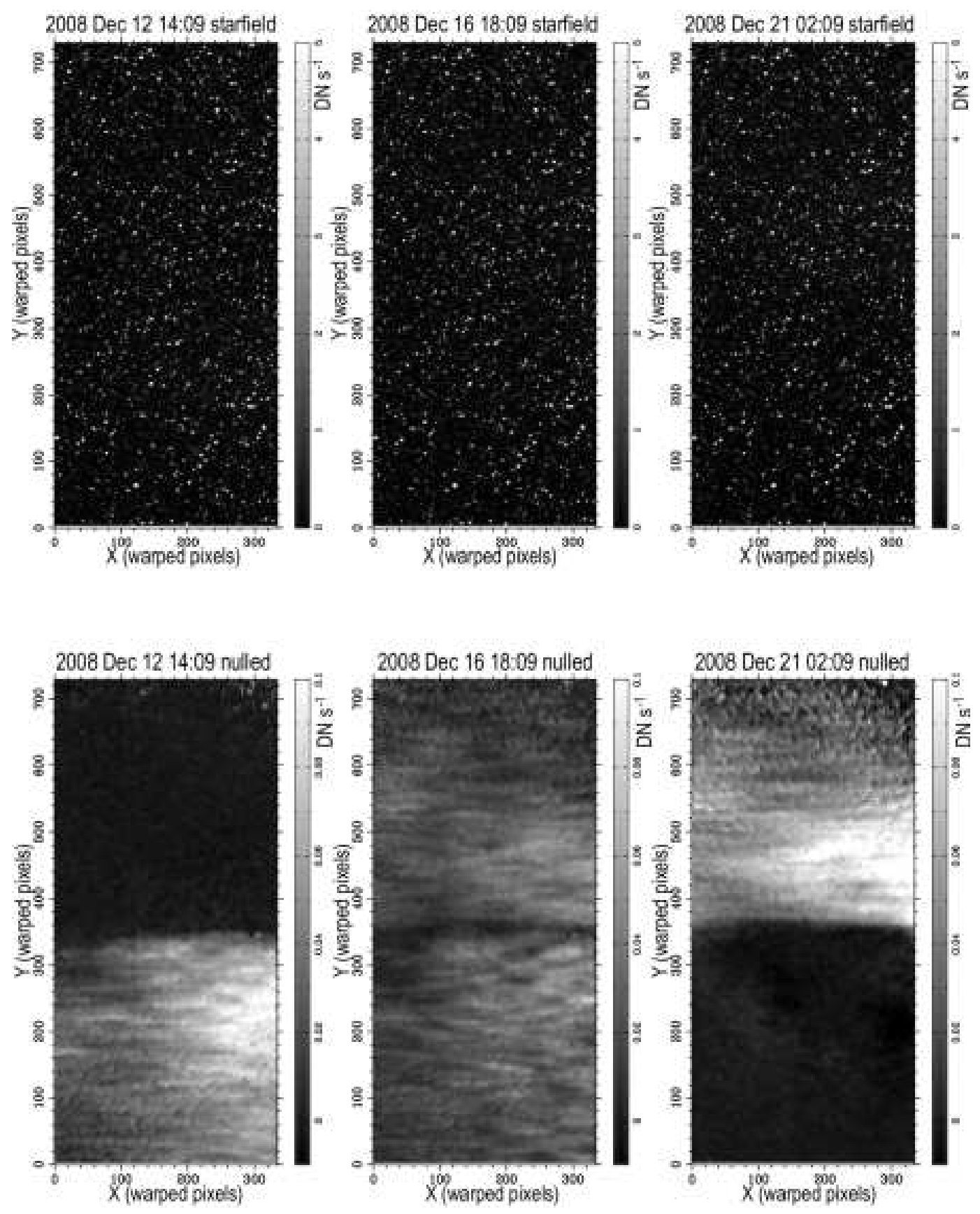

Fig. 7.- Freezing in the celestial coordinate system and starfield subtraction are demonstrated in these three images from the beginning (left), middle (middle), and end (right) of the dataset. Note factor-of-50 difference in the brightness scale between top (frozen) and bottom (frozen, starfield-subtracted) rows. Solar wind features are faintly visible but are obscured by residuum from the F coronal subtraction (see text). 

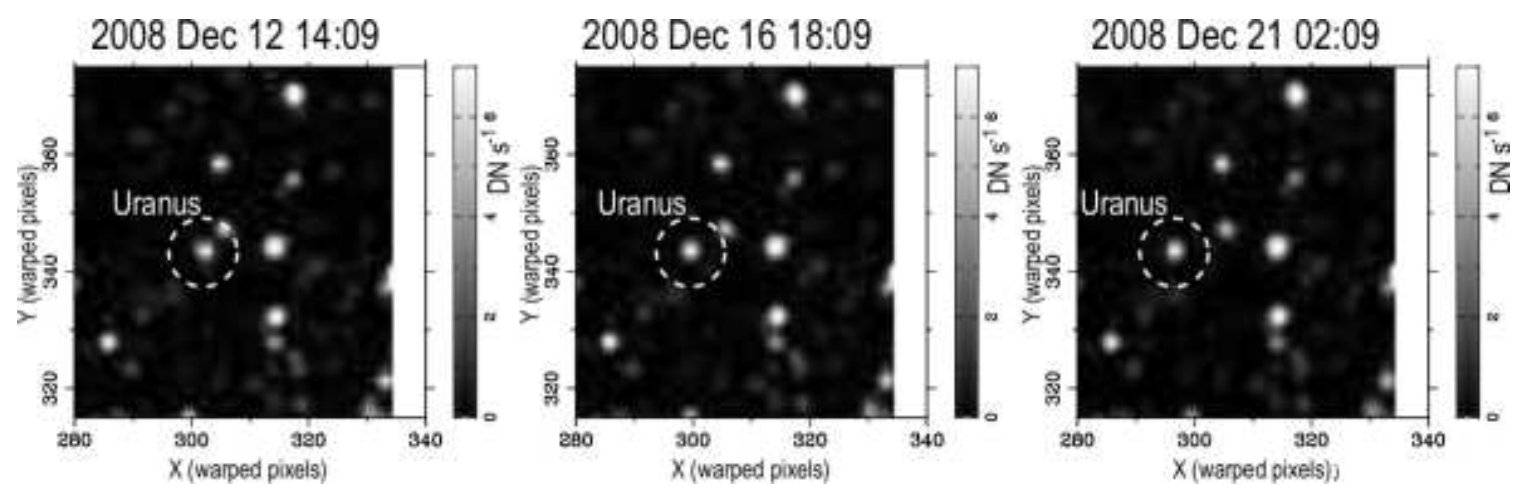

Fig. 8.- Uranus is visible in frozen starfield images from 2008 December. In these three panels, it is the only visible moving feature (circled and labeled), and has a central brightness of approximately $7 \mathrm{DN} \mathrm{s}^{-1}$.
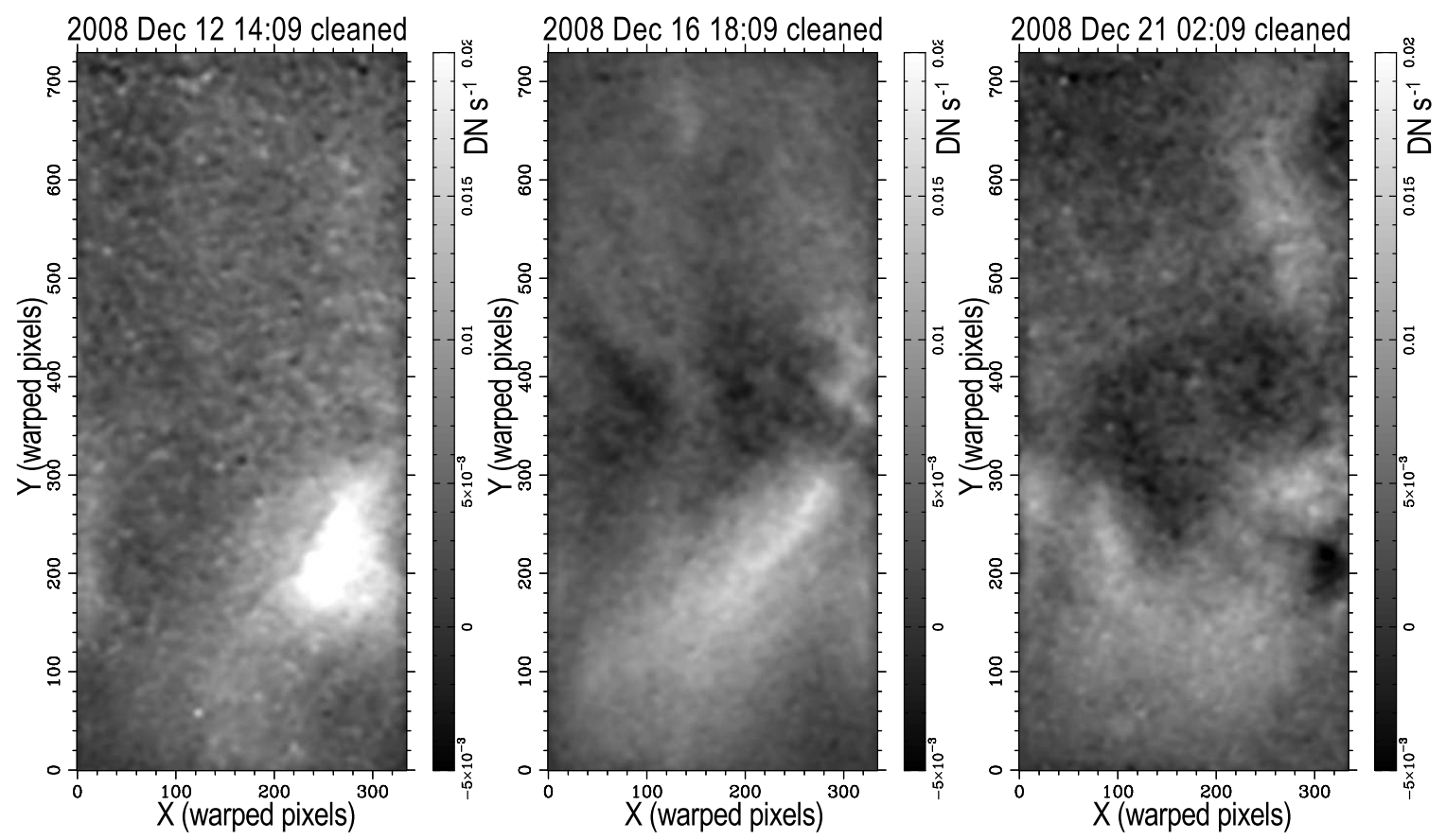

Fig. 9.- Cleaned images, in celestial coordinates, show solar wind at the $0.02 \mathrm{DN} \mathrm{s}^{-1}$ level (full scale), with discernible features an order of magnitude fainter still. The brightness scale has been reduced by a factor of 1000 from Figure 1, The noise level is about $1.5 \times 10^{-3} \mathrm{DN}$ $\mathrm{s}^{-1} \mathrm{RMS}$, or about $10^{-4}$ of the raw data amplitude. 

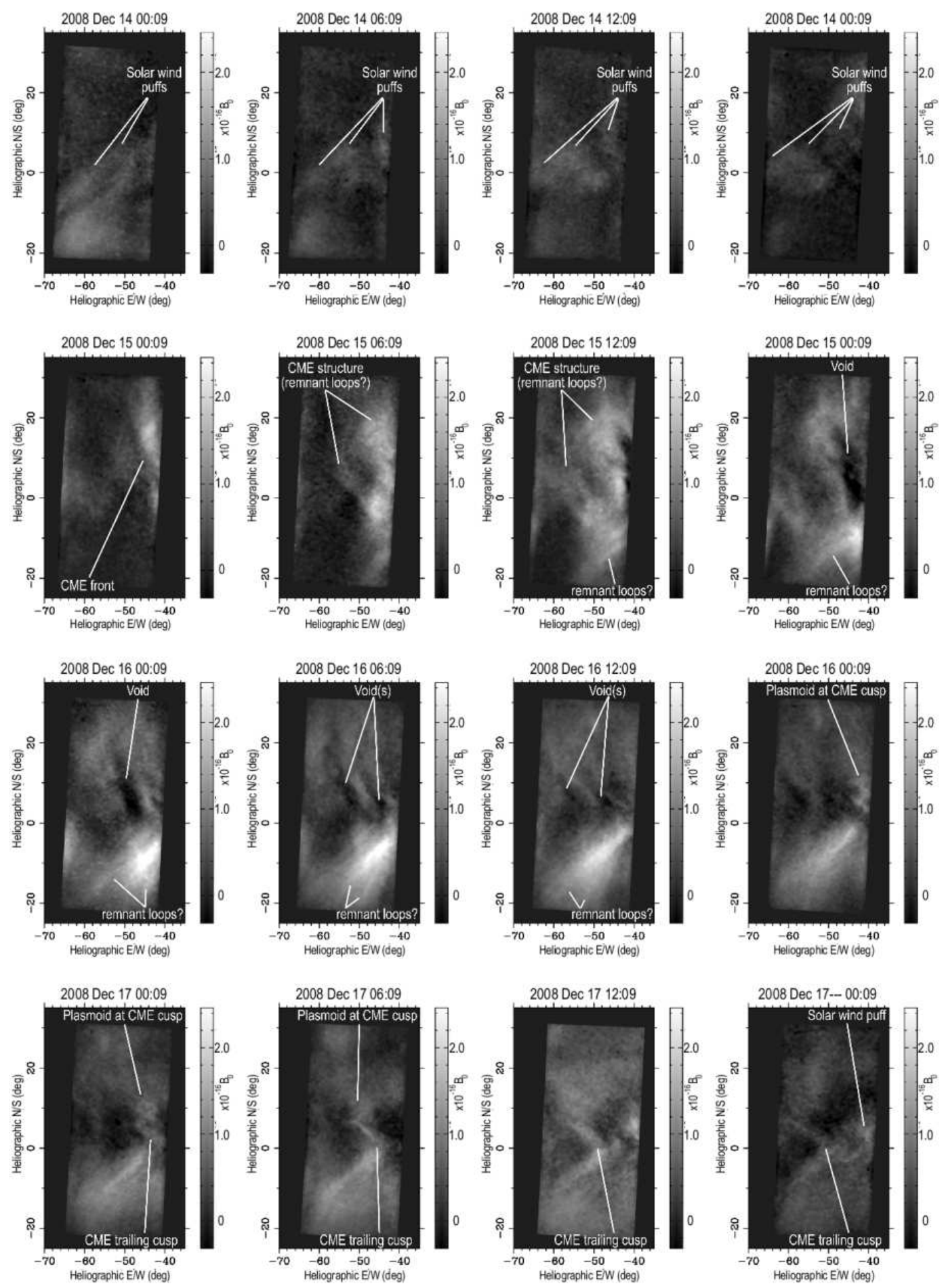

Fig. 10.- Background-subtracted HI-2 frames, in celestial coordinates, from 2008 December, show solar wind "puffs" and eddies, a bright CME front, post-front voids, and other related structures. The images have been sampled at 6 hour cadence and are a subset of the images in the attached movie file in the digital edition of this article. In both the images and the movie, the $\mathrm{S} / \mathrm{N}$ ratio of the brightest features is of order 30 , and faint features down to $3 \times 10^{-17} \mathrm{~B}_{\odot}$ are readily seen. 

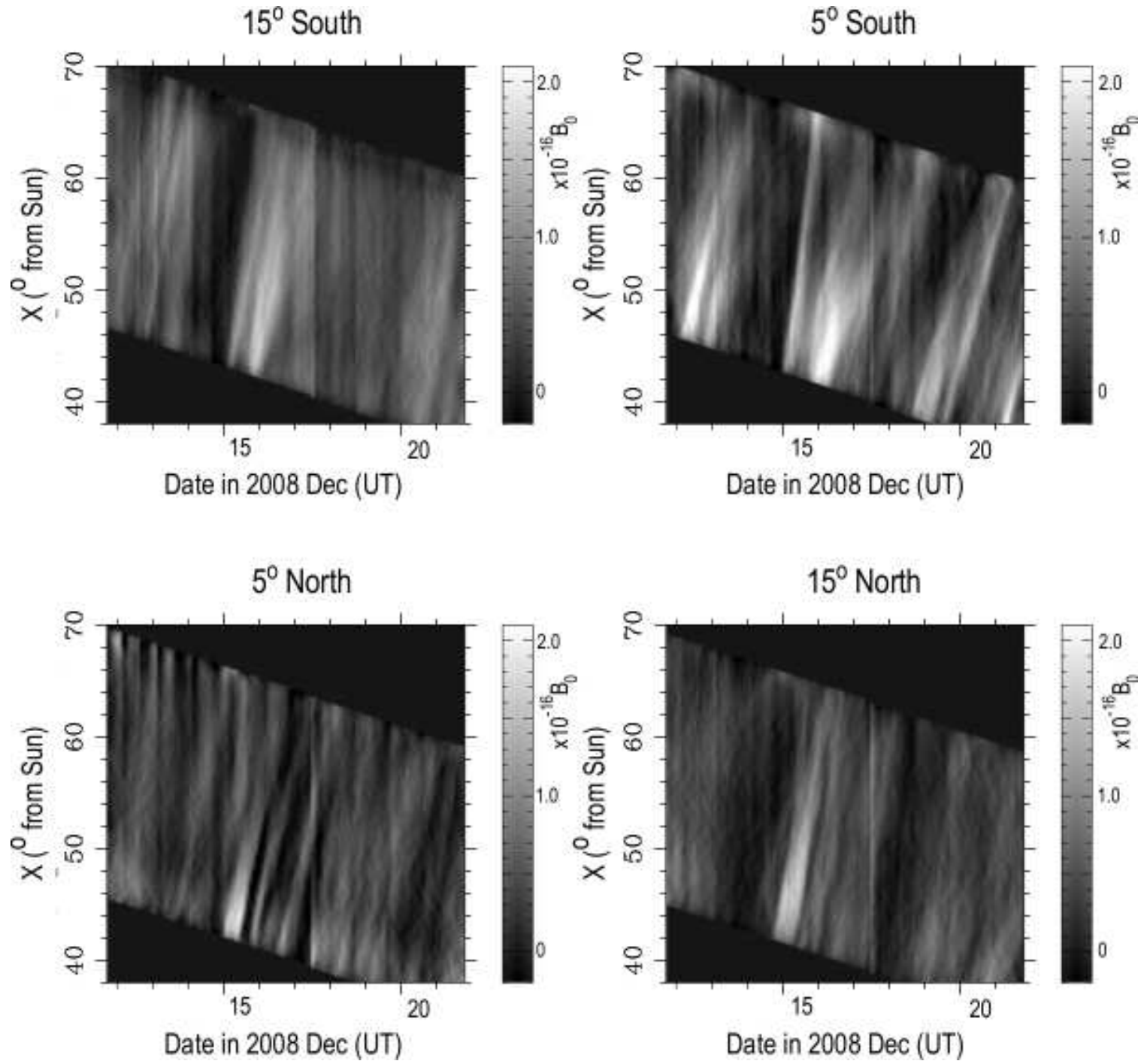

Fig. 11.- Elongation-time "J-plot" diagrams for the 11-day observation period in December 2008 showing multiple features. Each image represents a different position angle measured from the ecliptic plane: $15^{\circ}$ south (top left); $5^{\circ}$ south (top right); $5^{\circ}$ north (bottom left); $15^{\circ}$ north (bottom right). Only the regions subject to the advanced data processing are shown. 


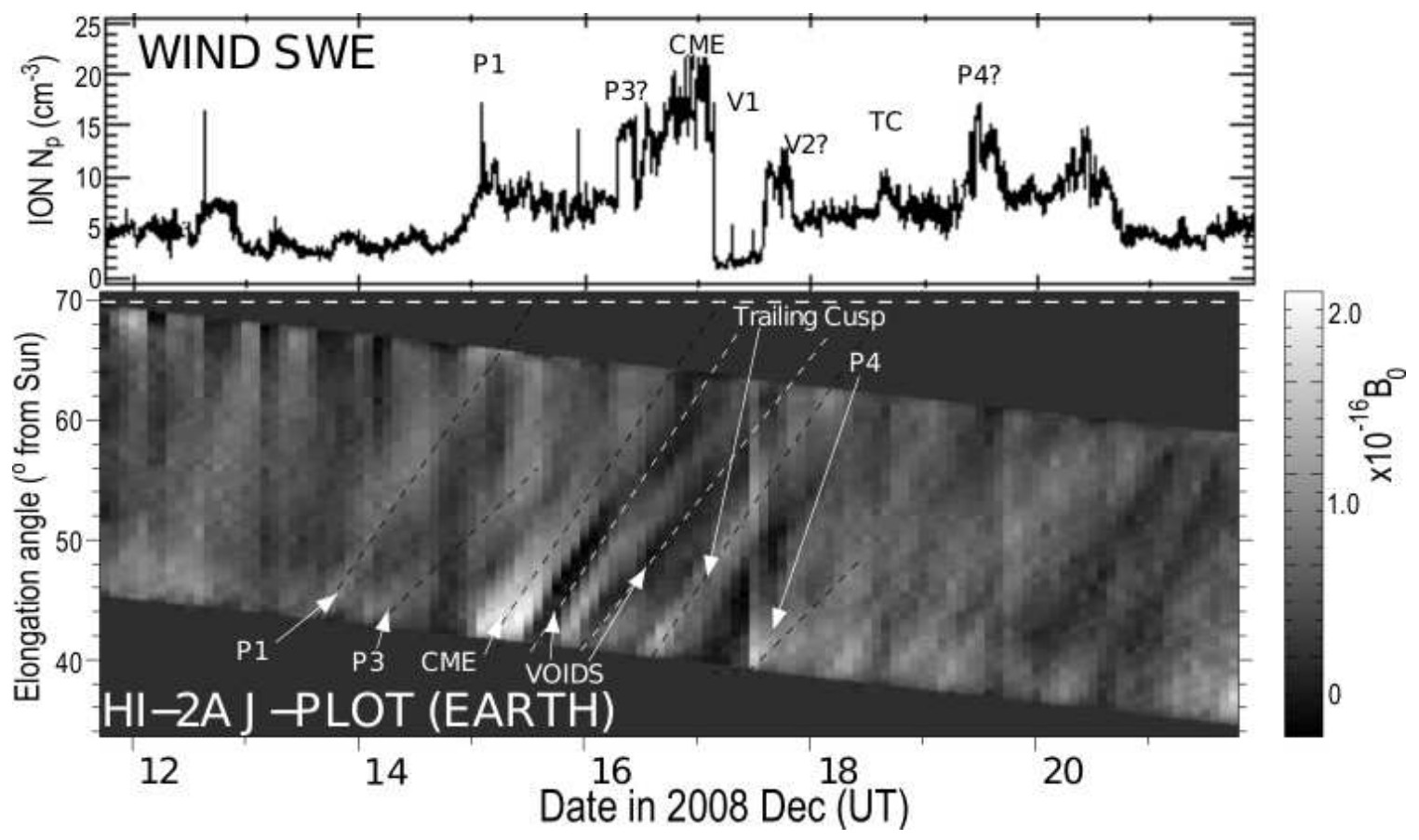

Fig. 12.- STEREO/HI-2A J-plot with in-situ density data provided by the Wind spacecraft for the same time period. These have been produced in the same manner as those in Figure 11. We have selected the position angle to be along the ecliptic, such that the Earth (and Venus) are intersected. The Earth, at $70^{\circ}$ elongation, is represented by a horizontal dashed line. 